\title{
Energy level alignment in Au/pentacene/PTCDA trilayer stacks
}

\author{
Parisa Sehati, Slawomir Braun and Mats Fahlman
}

\section{Linköping University Post Print}

\section{Tweet}

N.B.: When citing this work, cite the original article.

Original Publication:

Parisa Sehati, Slawomir Braun and Mats Fahlman, Energy level alignment in Au/pentacene/PTCDA trilayer stacks, 2013, Chemical Physics Letters, (583), 38-41.

http://dx.doi.org/10.1016/j.cplett.2013.07.035

Copyright: Elsevier

http://www.elsevier.com/

Postprint available at: Linköping University Electronic Press

http://urn.kb.se/resolve?urn=urn:nbn:se:liu:diva-81061 


\title{
Energy level alignment in Au/Pentacene/PTCDA trilayer stacks
}

\author{
P. Sehati, S. Braun and M. Fahlman \\ Department of Physics, Chemistry and Biology, Linköping University, 58183 Linköping, Sweden
}

\begin{abstract}
Ultraviolet Photoelectron Spectroscopy is used to investigate the energy level alignment and molecular orientation at the interfaces in Au/pentacene/PTCDA trilayer stacks. We deduced a standing orientation for pentacene grown on $\mathrm{Au}$ while we conclude a flat lying geometry for PTCDA grown onto pentacene. We propose that the rough surface of polycrystalline Au induces the standing geometry in pentacene. It is further shown that in-situ deposition of PTCDA on pentacene can influence the orientation of the surface pentacene layer, flipping part of the surface pentacene molecules into a flat lying geometry, maximizing the orbital interaction across the pentacenePTCDA heterojunction.
\end{abstract}

Keywords: Ultraviolet Photoelectron Spectroscopy (UPS), Orientation dependent Ionization Potential, Pentacene, PTCDA

\section{Introduction}

This study is aimed towards shedding light on the energy level alignment at Organic-Organic (OO) heterojunctions and factors impacting it, in particular intermolecular order effects. We selected the polycrystalline $\mathrm{Au} /$ pentacene/PTCDA system since pentacene and the perylene-derivative PTCDA (3, 4, 9, 10-perylene-tetracarboxylic dianhydride) long have served as "banana flies" for investigating the energy level alignment of $\pi$-conjugated organic molecules at well-defined surfaces [1-6]. Pentacene is of particular interest due to its ability to form (poly) crystalline thin films that leads to high hole-mobility values when used in e.g. Organic Thin Film Transistors (OTFTs) [7-9]. The quality of the crystalline pentacene film will significantly affect the obtained hole-mobility, and the crystallinity (and intermolecular order in general) of pentacene films depend on a number of parameters such as surface structure of the substrate, substrate temperature during film deposition, evaporation rate, etc. The details of the growth mechanism of monolayer and thin films of pentacene on different substrates hence have been extensively studied by many different experimental techniques as well as theoretical methods [9-30, 31 ]. In general, the strength of the interaction between the substrate and the molecule versus intermolecular interaction defines the orientation of the monolayer (ML). Strong intermolecular interaction and/or weak, negligible molecule- substrate interaction often will lead to edge-on or standing orientation at the interface 
while the opposite cases cause a flat or face-on configuration [13, 17, 32]. Pentacene and other aromatic molecules tend to have strong interaction (chemisorption) with clean metallic surfaces and this strong interaction leads to flat lying or face-on orientation of pentacene (and similar aromatic molecules), at least in the first few ML(s) [18, 20, 23, 24, 28, 33]. Additional film growth decouples the metal substrate and the bulk of the deposited molecules, which may lead to other orientations, e.g. standing. Aromatic molecules deposited on non-metallic surfaces and even metallic ones can grow also in standing orientation, however, already from the first few ML(s). One of the proposed explanations for the cause of this phenomenon is a rough surface of the substrate at the molecular scale which then can't provide well-defined adsorption sites [13]. Roughness prevents maximal interaction between substrate and adsorbate molecules and as a result, adsorbate molecules interact, overlap and couple with each other and provide a more stable configuration for themselves [9, 13, 25]. This highly coupled intermolecular packing often leads to (upright) standing or edge-on orientation. For example, physisorption of pentacene molecules on a non-metallic surface such as $\mathrm{SiO}_{2}$ which is used as gate dielectric in transistors leads to a standing orientation. Other examples of substrates which induce standing orientation in pentacene molecules are polycrystalline $\mathrm{Cu}$ [26], LSMO [21], PEDOT: PSS [16] and $\mathrm{C}_{60}$ [22].

That the substrate can have a strong influence on film order, especially at the interface, is thus well established. The interface effects on electronic properties, especially the energy level alignment, also are of importance, in particular to organic electronic devices. Indeed, as a result of contact between a $\pi$-conjugated molecule and another material, the electronic structure and parameters related to the electronic structure of the molecules at the interface can be modified through a variety of effects depending on the nature of the interface [34]. The Integer Charge Transfer (ICT) model [35-37] addresses energy level alignment at weakly interacting organic-organic and hybrid interfaces and hence is used in this study to interpret the experimental results. The model predicts that energy level alignment at such interfaces follows the Schottky-Mott regime or a Fermi-pinning regime depending on the position of the Fermi level and the oxidation/reduction energies of the molecules at the interface [35-37]. To wit, when the substrate work function is large enough to cause oxidation of molecules in the overlayer or small enough to promote reduction, charge transfer occurs and a dipole will be formed at the interface producing a vacuum level offset. The produced cation(s)/anion(s) after charge transfer process are called Integer Charge Transfer state(s): $\mathrm{ICT}^{+}$for a cation and $\mathrm{ICT}^{-}$for an anion. As long as the formation energy of the $\mathrm{ICT}^{+}$(cation) is smaller than the (dipole-modified) work function of the substrate then thermodynamically charge transfer and formation of the positive charge transfer state $\mathrm{ICT}^{+}$is favored and continues until the induced interface dipole is large enough equilibrate the Fermi level to the $\mathrm{ICT}^{+}$state. The opposite is true when we are dealing with the anions or negative charge transfer state ICT': when the energy gained 
by creating the $\mathrm{ICT}^{-}$is greater than the substrate work function, electron transfer from substrate to organic material takes place until equilibrium is achieved. If the substrate work function is situated in between the $\mathrm{ICT}^{+}$and $\mathrm{ICT}^{-}$states, no charge transfer occurs (barring defects) and Schottky-Mott type behavior is obtained [35-37]. As noted, in the Fermi level pinning regimes, the resulting work function upon interface completion does not directly depend on the substrate but is equal to the energy of the $\mathrm{ICT}^{+,-}$states, $\mathrm{E}_{\mathrm{ICT}+,-.}$ However, the $\mathrm{E}_{\mathrm{ICT}+,-}$ and hence the resulting work function may depend indirectly on the substrate as the $\mathrm{E}_{\mathrm{ICT}+,-}$ are affected by inter- and intra-molecular screening that in turn are affected by inter- and intra-molecular order [35, 36, 38, 39]. As discussed above, the molecule-substrate interaction during film formation strongly can affect the molecular order at the interface so as to make it radically different from the bulk (crystal) structure. An understanding of the molecular order (or lack thereof) at the interface is then useful to correctly predict and interpret energy level alignment at (hybrid) organic interfaces.

\section{Experimental}

Organic Molecular Beam Deposition (OMBD) technique performed using a Knudsen-type organic evaporator was employed to in situ grow thin films of the pentacene on the surface of chemically cleaned polycrystalline gold and subsequently in situ grow PTCDA films on the Au/pentacene substrates. The chemically cleaned gold surface ensured physisorption of the pentacene molecules and hence produced a weakly interacting interface, also the case for the pentacene/PTCDA interface. The evaporation rate of pentacene was roughly $3 \AA /$ min while PTCDA was evaporated with the rate of $\sim 2.5 \AA / m i n$. The substrates were kept at room temperature during deposition of pentacene and PTCDA as well as during measurements. Our home-made and designed photoelectron spectrometer has a base pressure lower than $10^{-9} \mathrm{mbar}$ and the photoemission experiments were carried out following each deposition step using monochromatized He I radiation with $21.2 \mathrm{eV}$ energy. The work function and dipole shifts were estimated from the secondary electron cut-off with an accuracy of $\pm 0.05 \mathrm{eV}$. The vertical ionization potential (IP) was measured with an accuracy of \pm 0.1 $\mathrm{eV}$ using the leading edge of the frontier valence feature. Intermolecular order directly affects the IP of molecular films as measured by photoelectron spectroscopy [40] and IP values hence can be used to determine the orientation of molecules in a layer [41]. We apply this technique to gain information of the intermolecular order of the pentacene and PTCDA films. 


\section{Results and discussions}

The evolution of the valence region photoemission spectrum of pentacene deposited in-situ on top of polycrystalline Au as a function of the thickness is shown in Fig. 1. At the left part of the figure secondary electron cut-off manifests the work function (WF) of the sample while in the right panel in the frontier occupied states region the energy difference between the low binding energy onset, here referred to as Valence Band Edge (VBE), added to the work function yields the vertical IP defined as IP $=\mathrm{WF}+\mathrm{VBE}$. The chemically cleaned gold surface has a WF of $\sim 4.6 \mathrm{eV}$, a typical WF value for gold handled in ambient air [42]. At $2.7 \mathrm{~nm}$ film thickness, the pentacene features can be seen clearly and the Au peak is completely suppressed. The observation that such a thick film of pentacene is necessary in order to suppress the gold signal indicates the organic film (initially) grows as islands on polycrystalline Au, likely a combination of roughness of the Au substrate, weak interaction between the gold surface and the pentacene molecules (physisorption) and a strong intermolecular coupling [32]. The IP is almost constant for the different pentacene film thicknesses while the WF spans over a range from $4.46 \mathrm{eV}$ at $2.7 \mathrm{~nm}$ to $4.35 \mathrm{eV}$ at $7.1 \mathrm{~nm}$ thickness. These results are collected in Table 1. The decrease in WF upon pentacene deposition is attributed to charge transfer from pentacene molecules to the gold substrate causing pentacene cations to be formed and the Fermi level is pinned to the $\mathrm{E}_{\mathrm{ICT}+}$ of the pentacene molecules. The IP and WF values are in line with those previously reported [21, 22], where a standing geometry for the pentacene molecules at weakly-interacting interfaces was found.

The $71 \AA$ adlayer of pentacene on polycrystalline Au was subsequently used as template to in-situ deposition of PTCDA films. The film thickness dependent evolution of the electronic structure of PTCDA-on-pentacene was monitored by UPS. Upon PTCDA deposition an increase in the work function occurred, saturating at $\sim 4.6 \mathrm{eV}$, resulting in an overall vacuum level shift of $\sim 0.25 \mathrm{eV}$, see Table 2. This is in good agreement with the theoretical prediction of $4.7 \mathrm{eV}$ for the $\mathrm{E}_{\text {ICT- }}$ value of face-on ordered PTCDA [38, 39], suggesting electron transfer from pentacene to PTCDA, creating PCTDA anions and pinning the Fermi level to the $\mathrm{E}_{\mathrm{ICT}}$ state of PTCDA. The work function and IP values for the PTCDA films also are in good agreement with the case of PTCDA on Au(111) where PCTDA forms face-on ordered layers [2], supporting the proposed scenario. Following the evolution of the frontier occupied electronic structure in Fig. 2, we note that the frontier peak of pentacene (related to the highest occupied molecular orbital, HOMO), located at $\sim 1 \mathrm{eV}$, remains visible up to almost $2 \mathrm{~nm}$ of PTCDA thickness, suggesting (initial) island-type growth of the PTCDA overlayer likely caused by roughness of the pentacene surface. Another prominent peak in this figure is frontier peak (HOMO) of the deposited PTCDA film located at $\sim 2.8 \mathrm{eV}$, a position that roughly coincides with deeper lying pentacene features, see bottom spectrum of Fig. 2 . After 3 $\mathrm{nm}$ thickness, the pentacene HOMO feature at $\sim 1 \mathrm{eV}$ has disappeared but is replaced by a new weak 
feature located at $\sim 1.5 \mathrm{eV}$. The origin of this peak is unclear. One possible scenario is to attribute the peak to the partial filling, due to charge transfer from pentacene, of the lowest unoccupied molecular orbital of PTCDA, which is then pulled down below Fermi level. We propose that this explanation should be excluded since the new peak is located at $1.5 \mathrm{eV}$, which is far too deep to be the singly-occupied former LUMO of PTCDA, typical values being around 0.1-0.5 eV. Also, the experimental sequence $\mathrm{Au} /$ pentacene/PTCDA was repeated several times and though the WF and IP evolution was the same, the new feature did not always appear, see a different series displayed in Fig. 3. We note instead that the IP of face-on lying pentacene is $0.55 \mathrm{eV}$ higher than that of standing pentacene [43], a value identical to the binding energy difference between the original pentacene HOMO and the new feature that appears in Fig. 2, suggesting that the disappearance of the frontier peak and creation of the new feature is due to pentacene molecules at the PTCDA interface "flipping" from a standing orientation into a lying orientation in order to maximize $\pi-\pi$ interaction between the pentacene and PTCDA molecules as well as maximize hydrogen bonding between the pentacene hydrogen and PTCDA oxygen at the interface. However, converting from a standing to lying orientation also costs energy, with larger more well-ordered domains/islands of pentacene at the surface being more costly to convert than a rougher surface featuring smaller less-ordered domains/islands. Hence, for sufficiently well-ordered and flat surfaces of pentacene, the cost of converting the surface into a lying order is larger than energy gained from increased molecular interaction with PTCDA and the peak at $0.55 \mathrm{eV}$ higher binding energy will not appear, whereas for sufficiently disordered and rough surfaces of pentacene, the cost of converting the surface into a lying order is smaller than energy gained from increased molecular interaction with PTCDA and the peak at $0.55 \mathrm{eV}$ higher binding energy will appear. Finally, there will be pentacene films where parts of the surface will be converted and other parts not, depending on local variations in order and roughness, see Fig. 4, which is likely the most realistic scenario under our growth conditions. Indeed, molecular dynamics simulations of the growth of flat-lying PTCDA on standing pentacene interfaces show that a thin disorder interface region is created even for a "perfect" starting pentacene surface [44].

\section{Summary}

We studied energy level alignment in the model system of polycrystalline Au/pentacene/PTCDA. UPS measurements showed a $\sim 0.3 \mathrm{eV}$ downward dipole at Au/pentacene interface and a $\sim 0.25 \mathrm{eV}$ upward dipole at pentacene/PTCDA interface due to Fermi level pinning to the pentacene $\mathrm{ICT}^{+}$and PTCDA ICT ${ }^{-}$state, respectively. In this trilayer system, pentacene is a global donor: it donates electrons to both the Au substrate and the PTCDA overlayer. Our results further suggest a standing geometry for pentacene molecules on $\mathrm{Au}$, in line with previous studies of rough weakly-interacting 
surfaces, and a face-on geometry for the PTCDA molecules deposited onto the pentacene films. We noted that though the evolution of IP and WF with thickness was consistent for all of the experimental series, we obtained a variation in the frontier electronic structure for the pentacene/PTCDA interface where a new feature sometimes appeared upon formation of the PTCDA overlayer. We attribute the new feature to a change in the inter-molecular order of pentacene molecules at the interface from standing to lying. We propose that the change in orientation and the variations in the extent of the effect seen from series to series can be explained by the interplay between energy gained in PTCDA-pentacene interaction at the interface from faceon - face-on order and energy lost flipping domains of pentacene molecules from standing to lying orientation. Such a reorientation is likely to have a profound effect on e.g. charge transfer across the heterojunction.

\section{Acknowledgement}

We acknowledge funding from the European Community's Framework Programme under grant no. FP7-NMP-228424 of the MINOTOR project as well as a project grant from the Swedish Energy Agency, STEM.

\section{References}

[1] W. Chen, D.C. Qi, Y.L. Huang, H. Huang, Y.Z. Wang, S. Chen, X.Y. Gao, and A.T.S. Wee, J. Phys. Chem. C, 113 (2009) 12832.

[2] S. Duhm, A. Gerlach, I. Salzmann, B. Bröcker, R.L. Johnson, F. Schreiber, and N. Koch, Org. Electron., 9 (2008) 111.

[3] N. Nicoara, E. Román, J.M. Gómez-Rodríguez, J.A.M.-. Gago, and J. Méndez, Org. Electron., 7 (2006) 287.

[4] T. Wagner, A. Bannani, C. Bobisch, H. Karacuban, and R. Möller, J. Phys. Condens. Mat., 19 (2007) 056009.

[5] Y. Zou, L. Kilian, A. Schöll, T. Schmidt, R. Fink, and E. Umbach, Surf. Sci., 600 (2006) 1240.

[6] R. Ruiz, B. Nickel, N. Koch, L.C. Feldman, R.F. Haglund, A. Kahn, F. Family, and G. Scoles, Phys. Rev. Lett., 91 (2003) 4.

[7] C.D. Dimitrakopoulos, A.R. Brown, and A. Pomp, J. Appl. Phys. , 80 (1996) 2501.

[8] C.D. Dimitrakopoulos and P.R.L. Malenfant, Adv. Mater., 14 (2002) 99.

[9] M. Shtein, J. Mapel, J.B. Benziger, and S.R. Forrest, Appl. Phys. Lett., 81 (2002) 268.

[10] C.B. France, P.G. Schroeder, J.C. Forsythe, and B.A. Parkinson, Langmuir, 19 (2003) 1274.

[11] C.B. France, P.G. Schroeder, and B.A. Parkinson, Nano Lett., 2 (2002) 693.

[12] H. Fukagawa, S. Kera, T. Kataoka, S. Hosoumi, Y. Watanabe, K. Kudo, and N. Ueno, Adv.Mater., 19 (2007) 665.

[13] H. Fukagawa, H. Yamane, T. Kataoka, S. Kera, M. Nakamura, K. Kudo, and N. Ueno, Phys. Rev. B, 73 (2006) 245310.

[14] P. Guaino, D. Carty, G. Hughes, O. McDonald, and A.A. Cafolla, Appl. Phys. Lett., 85 (2004) 2777.

[15] N. Koch, A. Elschner, J.P. Rabe, and R.L. Johnson, Adv. Mater., 17 (2005) 330.

[16] N. Koch, A. Elschner, J. Schwartz, and A. Kahn, Appl. Phys. Lett., 82 (2003) 2281. 
[17] N. Koch, A. Kahn, J. Ghijsen, J.-J. Pireaux, J. Schwartz, R.L. Johnson, and A. Elschner, Appl. Phys. Lett., 82 (2003) 70.

[18] N. Koch, I. Salzmann, R.L. Johnson, J. Pflaum, R. Friedlein, and J.P. Rabe, Org. Electron., 7 (2006) 537.

[19] N. Koch, A. Vollmer, I. Salzmann, B. Nickel, H. Weiss, and J.P. Rabe, Phys.Rev.Lett., 96 (2006) 156803.

[20] D. Käfer, L. Ruppel, and G. Witte, Phys. Rev. B, 75 (2007) 085309.

[21] F.H. Li, P. Graziosi, Q. Tang, Y.Q. Zhan, X.J. Liu, V. Dediu, and M. Fahlman, Phys. Rev. B, 81 (2010) 6.

[22] X. Liu, Y. Zhan, S. Braun, F. Li, and M. Fahlman, Phys. Rev. B, 80 (2009) 115401.

[23] S. Lukas, S. Söhnchen, G. Witte, and C. Wöll, ChemPhysChem. , 5 (2004) 266.

[24] S. Lukas, G. Witte, and C. Wöll, Phys. Rev. Lett., 88 (2002) 028301.

[25] D. Nabok, P. Puschnig, C. Ambrosch-Draxl, O. Werzer, R. Resel, and D.-M. Smilgies, Phys. Rev. B, 76 (2007) 235322.

[26] M. Oehzelt, R. Resel, C. Suess, R. Friedlein, and W.R. Salaneck, J. Chem. Phys., 124 (2006) 054711.

[27] R. Ruiz, B. Nickel, N. Koch, L.C. Feldman, R.F. Haglund, A. Kahn, and G. Scoles, Phys. Rev. B, 67 (2003) 125406.

[28] P.G. Schroeder, C.B. France, J.B. Park, and B.A. Parkinson, J. Appl. Phys., 91 (2002) 3010.

[29] P.G. Schroeder, C.B. France, J.B. Park, and B.A. Parkinson, J. Phys. Chem. B, 107 (2003) 2253.

[30] S. Söhnchen, S. Lukas, and G. Witte, J. Chem. Phys., 121 (2004) 525.

[31] S.L. Wong, H. Huang, Y.L. Huang, Y.Z. Wang, X.Y. Gao, T. Suzuki, W. Chen, and A.T.S. Wee, J. Phys. Chem. C, 114 (2010) 9356.

[32] S. Kowarik, A. Gerlach, and F. Schreiber, J. Phys. Cond. Matter, 20 (2008) 184005.

[33] P. Ruffieux, O. Gröning, M. Bielmann, C. Simpson, K. Müllen, L. Schlapbach, and P. Gröning, Phys. Rev. B, 66 (2002) 073409.

[34] H. Ishii, K. Sugiyama, E. Ito, and K. Seki, Adv. Mater., 11 (1999) 605.

[35] S. Braun, W.R. Salaneck, and M. Fahlman, Adv. Mater., 21 (2009) 1450.

[36] M. Fahlman, A. Crispin, X. Crispin, S.K.M. Henze, M.P.d. Jong, W. Osikowicz, C. Tengstedt, and W.R. Salaneck, J. Phys.: Condens. Matter, 19 (2007) 183202.

[37] C. Tengstedt, W. Osikowicz, W.R. Salaneck, I.D. Parker, C.-H. Hsu, and M. Fahlman, Appl. Phys. Lett., 88 (2006) 053502.

[38] M. Bokdam, D. Cakir, and G. Brocks, Appl. Phys. Lett., 98 (2011) 113303.

[39] D. Çakir, M. Bokdam, M.P.d. Jong, M. Fahlman, and G. Brocks, Appl. Phys. Lett., 100 (2012) 203302.

[40] W.R. Salaneck, Phys. Rev. Lett., 40 (1978) 60.

[41] S. Duhm, G. Heimel, I. Salzmann, H. Glowatzki, R.L. Johnson, A. Vollmer, J.P. Rabe, and N. Koch, Nature Mater., 7 (2008) 326.

[42] W. Osikowicz, M.P. de Jong, S. Braun, C. Tengstedt, M. Fahlman, and W.R. Salaneck, Appl. Phys. Lett., 88 (2006) 3.

[43] I. Salzmann, S. Duhm, G. Heimel, M. Oehzelt, R. Kniprath, R.L. Johnson, J.P. Rabe, and N. Koch, J. Am. Chem. Soc., 130 (2008) 12870.

[44] A. Poschlad, V. Meded, R. Maul, and W. Wenzel, Nanoscale Res. Lett., 7 (2012) 1.

\section{Table and Figure Captions}

Table 1. Thickness evolution of the UPS-measured work function (WF) and vertical ionization potential (IP) of pentacene film on Au. 
Table 2. Thickness evolution of the UPS-measured work function (WF) of PTCDA on pentacene.

Figure 1. Thickness-dependent evolution of (left panel) work function and (right panel) valence electronic structure of pentacene in situ grown on a polycrystalline gold substrate.

Figure 2. Thickness-dependent evolution of frontier valence electronic structure of PTCDA in situ grown on a pentacene-on-gold substrate.

Figure 3. Thickness-dependent evolution of frontier valence electronic structure of PTCDA in situ grown on a pentacene-on-gold substrate (different series).

Figure 4. Schematic picture of Au/pentacene/PTCDA multilayer stack. 
Table 1.

\begin{tabular}{|c|c|c|c|}
\hline & Thickness $(\AA)$ & WF $(\mathrm{eV})$ & $\mathrm{IP}(\mathrm{eV})$ \\
\hline \multirow{2}{*}{$\mathrm{Au}$} & - & 4.63 & 4.63 \\
\hline \multirow{2}{*}{ Pentacene } & 27 & 4.46 & 4.78 \\
\cline { 2 - 4 } & 49 & 4.42 & 4.85 \\
\cline { 2 - 4 } & 71 & 4.35 & 4.83 \\
\hline
\end{tabular}


Table 2

\begin{tabular}{|l|c|c|}
\hline \multirow{4}{*}{ Pentacene } & Thickness $(\AA)$ & WF $(\mathrm{eV})$ \\
\hline \multirow{4}{*}{ PTCDA } & 41 & 4.35 \\
\cline { 2 - 3 } & 7 & 4.41 \\
\cline { 2 - 3 } & 10 & 4.44 \\
\cline { 2 - 3 } & 13 & 4.45 \\
\cline { 2 - 3 } & 19 & 4.52 \\
\cline { 2 - 3 } & 30 & 4.57 \\
\cline { 2 - 3 } & 45 & 4.57 \\
\hline
\end{tabular}




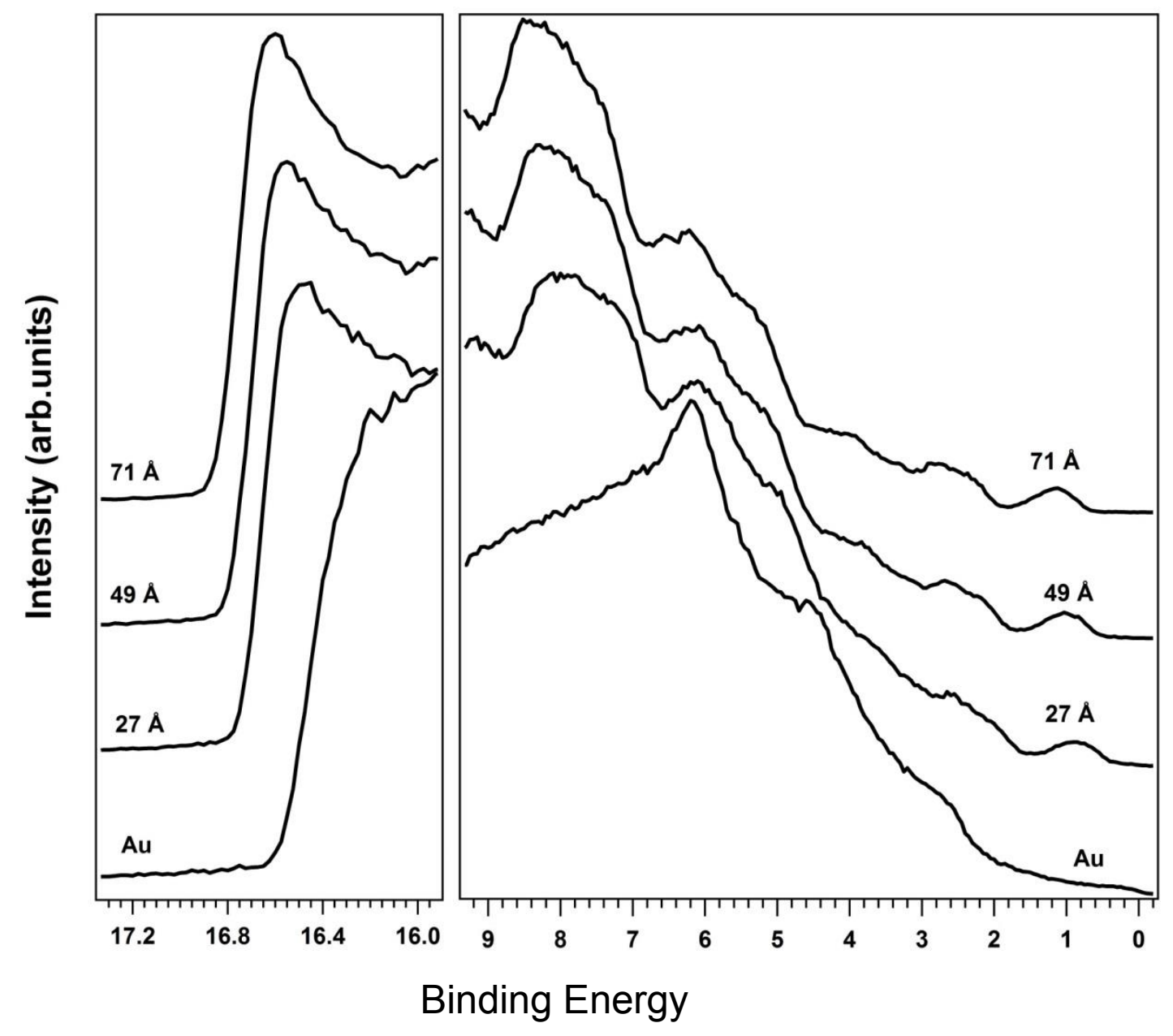

Figure 1. 


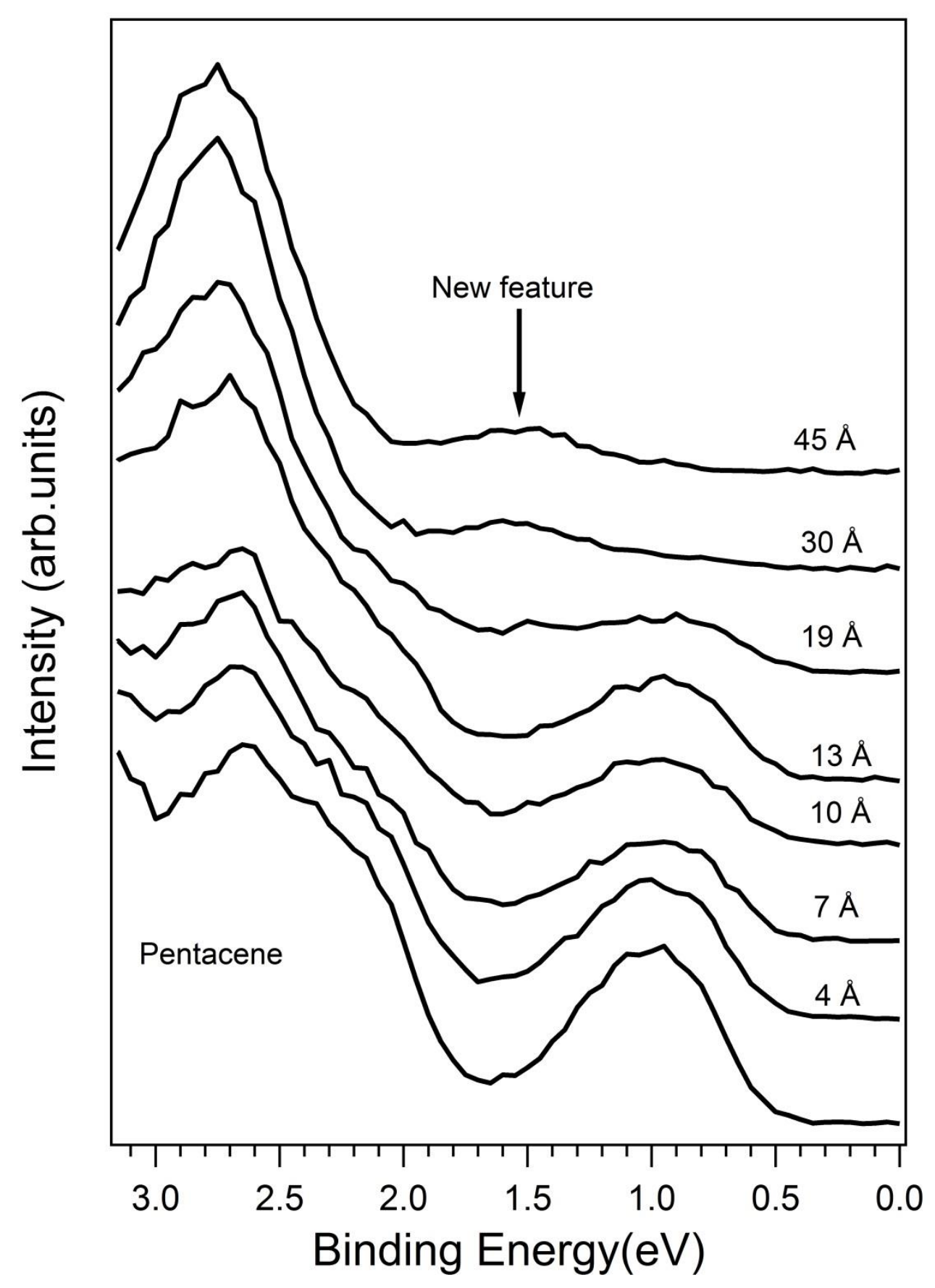

Figure 2. 


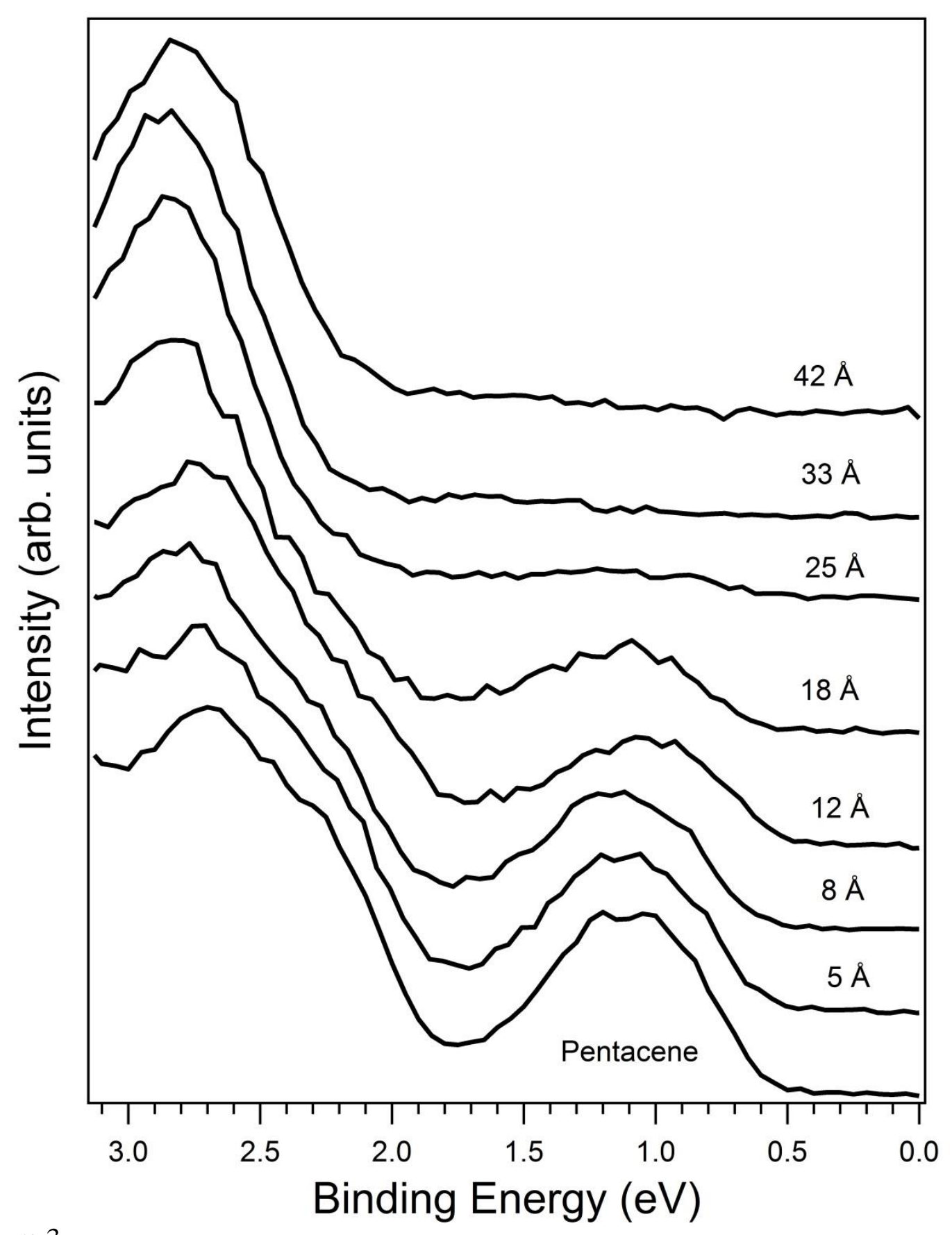

Figure 3. 


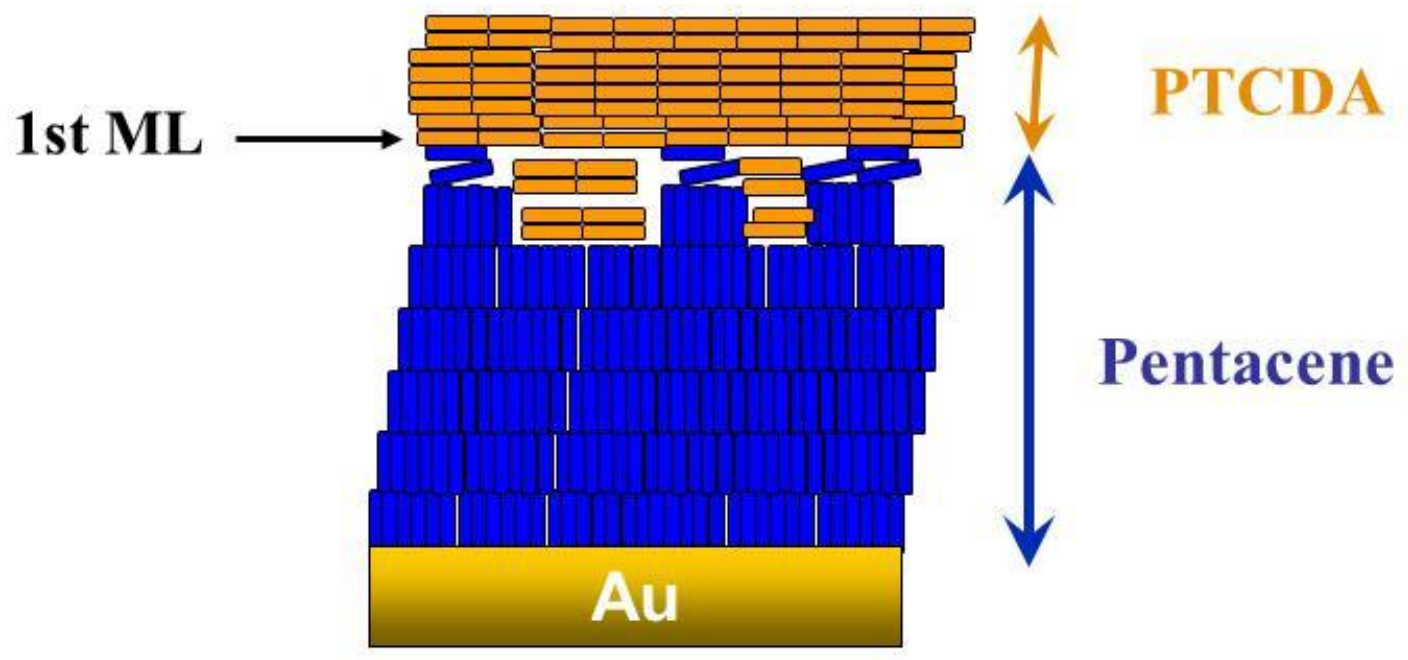

Figure 4.

(Color on web, black-and-white in publication) 